\title{
Pediatric cerebral malaria: a scourge of Africa
}

\section{Douglas G Postels*1, Yamikani F Chimalizeni², Macpherson Mallewa², Michael J Boivin' \& Karl B Seydel' \\ 'Michigan State University, East Lansing, MI 48824, USA \\ 2Department of Pediatrics, University of Malawi College of Medicine, Private Bag 360, Blantyre 3, Malawi \\ *Author for correspondence: Tel.: +7 5178840276 = Fax: +7 5178840275 - douglas.postels@ht.msu.edu}

Cerebral malaria, defined as an otherwise unexplained coma in a patient with Plasmodium falciparum parasitemia, affects up to 1 million people per year, the vast majority of them being children living in sub-Saharan Africa. Despite optimal treatment, this condition kills $15 \%$ of those affected and leaves $30 \%$ of survivors with neurologic sequelae. The clinical diagnosis is hampered by its poor specificity, but the presence or absence of a malarial retinopathy in cerebral malaria has proven to be important in the differentiation of underlying coma etiology. Both antimalarials and intense supportive care are necessary for optimal treatment. As of yet, clinical trials of adjunctive therapies have not improved the high rates of mortality and morbidity. Survivors are at high risk of neurologic sequelae including epilepsy, neurodisabilities and cognitive-behavioral problems. The neuroanatomic and functional bases of these sequelae are being elucidated. Although adjunctive therapy trials continue, the best hope for African children may lie in disease prevention. Strategies include bednets, chemoprophylaxis and vaccine development.

\section{Medscape Medscape: Continuing Medical Education Online}

This activity has been planned and implemented in accordance with the Essential Areas and policies of the Accreditation Council for Continuing Medical Education through the joint sponsorship of Medscape, LLC and Future Medicine Ltd. Medscape, LLC is accredited by the ACCME to provide continuing medical education for physicians.

Medscape, LLC designates this journal-based CME activity for a maximum of 1 AMA PRA Category 1 Credit(s) ${ }^{\mathrm{TM}}$. Physicians should claim only the credit commensurate with the extent of their participation in the activity.

All other clinicians completing this activity will be issued a certificate of participation. To participate in this journal CME activity: (1) review the learning objectives and author disclosures; (2) study the education content; (3) take the post-test with a $70 \%$ minimum passing score and complete the evaluation at www.medscape.org/journal/fnl; (4) view/print certificate.

Release date: 14 December 2012; Expiration date: 14 December 2013

\section{Learning objectives}

Upon completion of this activity, participants should be able to:

- Describe diagnosis and outcomes of pediatric CM, based on a review

- Describe management of pediatric CM, based on a review

- Describe the epidemiology and prevention of pediatric CM, based on a review
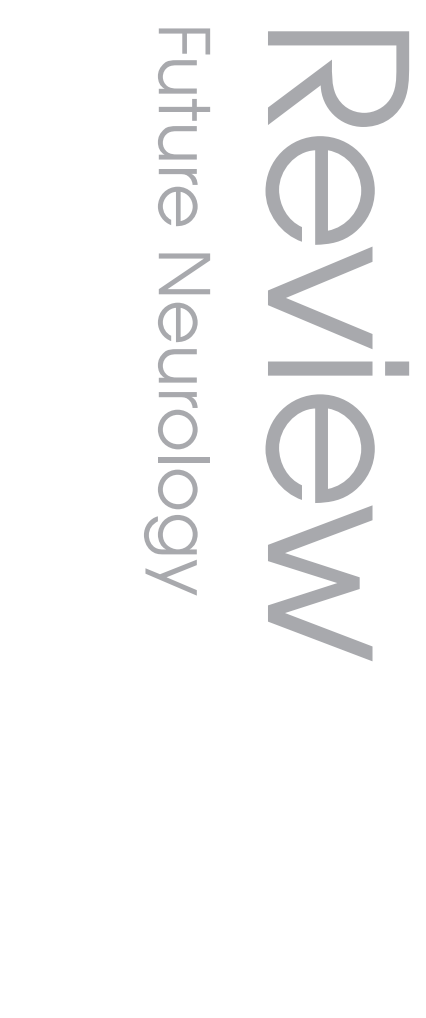
Financial \& competing interests disclosure

Editor: Elisa Manzotti, Publisher, Future Science Group. Disclosure: Elisa Manzotti has disclosed no relevant financial relationships.

CME author: Laurie Barclay, Freelance writer and reviewer, Medscape, LLC. Disclosure: Laurie Barclay, MD, has disclosed no relevant financial relationships.

Authors \& credentials: Douglas G Postels, Michigan State University, East Lansing, MI 48824, USA. Disclosure: Douglas $G$ Postels has disclosed no relevant financial relationships. Yamikani F Chimalizeni, Department of Pediatrics, University of Malawi College of Medicine, Private Bag 360, Blantyre 3, Malawi. Disclosure: Yamikani F Chimalizeni has disclosed no relevant financial relationships. Macpherson Mallewa, Department of Pediatrics, University of Malawi College of Medicine, Private Bag 360, Blantyre 3, Malawi. Disclosure: Macpherson Mallewa has disclosed no relevant financial relationships. Michael J Boivin, Michigan State University, East Lansing, MI 48824, USA. Disclosure: Michael Boivin has disclosed no relevant financial relationships. Karl B Seydel, Michigan State University, East Lansing, MI 48824, USA. Disclosure: Karl B Seydel has disclosed no relevant financial relationships.

No writing assistance was utilized in the production of this manuscript.

Cerebral malaria (CM) is defined as an otherwise unexplained coma in a patient with Plasmodium falciparum parasitemia. With approximately 3.3 billion people at risk and 216 million clinical episodes annually, malaria is the most important parasitic disease of mankind [1]. CM is one of the major complications of malaria and a leading contributor to death rates. The majority of deaths from $\mathrm{CM}$ are among children living in subSaharan Africa. Rates of neurologic morbidity in survivors are high. Although research continues, progress has been slow owing to the lack of an adequate animal model. The information presented in this review is derived primarily from studies of CM in African children, although occasionally research results derived from evaluations of adult patients are included. As disease manifestations are significantly different between adults and children these instances are mentioned within the text. Although severe malaria and CM are usually caused by $P$. falciparum, severe disease caused by infection with Plasmodium vivax has been increasingly reported in Asia, South America and Africa in the past several years [2-5]. This review will focus on $P$. falciparum; reviews on the emerging possibility of $P$. vivax $\mathrm{CM}$ are available elsewhere [6].

\section{Worldwide burden of disease}

The epidemiology of malaria, severe malaria and $\mathrm{CM}$ is changing. Many countries are registering reductions in malaria incidence and there has been a $25 \%$ reduction in worldwide mortality from the disease since 2000 [1]. Despite these encouraging figures, malaria remains a major cause of mortality and morbidity in many areas of the world, with an estimated 655,000 deaths in 2010 . A total of $86 \%$ of these victims were children under the age of 5 years old and 91\% of malaria deaths occurred in Africa [1].
Plasmodium falciparum is the most common cause of severe malaria. The descriptor 'severe' incorporates all types of malaria that may lead to death. Severe malaria is characterized by severe anemia, prostration, repeated seizures, impaired consciousness, hypoglycemia, respiratory distress or metabolic acidosis. CM is a common form of severe malaria and, along with severe malaria anemia, is one of the major contributors to malaria mortality. Without treatment, the disease is universally fatal. Despite optimal treatment, mortality is $15-22 \%$ [7-9]. Survivors do not escape unscathed; cohort studies show that approximately one-third of CM survivors have persistent neurologic sequelae $[10,11]$.

\section{Pathogenesis}

The precise mechanisms of CM pathogenesis remain unclear, but appear to be a combination of mechanical obstruction of the cerebral vasculature and intravascular inflammation. Sequestration of parasitized erythrocytes in cerebral vasculature is likely central to the process $[12,13]$. In the majority of patients who have died with clinically defined CM (see the section entitled 'Diagnosis' below), this irreversible attachment of parasitized erythrocytes to vascular endothelium is prominent in the brain [14]. Sequestration is visible during life in some $\mathrm{CM}$ patients as filling defects or mottling of retinal vessels [15]. That sequestered parasitized erythrocytes are present in cerebral vasculature in all patients with CM is a conjecture inferred from autopsy studies. Sequestration is mediated by a well-characterized family of proteins expressed on parasitized erythrocytes. The most important of these is PfEMP1. Genes encoding this protein, termed var, are highly variant but can be grouped into five families based on promoter region. Emerging evidence 
suggests that the type of infecting var gene family is associated with disease severity [16-18]. Host endothelial proteins binding to PfEMP1 and mediating sequestration are less well defined. In vitro experiments have demonstrated parasitized erythrocyte interactions with more than ten host endothelial cell proteins [19]. In vivo, the relative importance of each of these erythrocyte-endothelial interactions is more difficult to ascertain. The distribution of any of these candidate endothelial receptors does not fully explain the organ distribution of sequestration. Whether combinations of receptors, novel receptors or a unique mechanical or biological milieu in the cerebral vasculature explains sequestration patterns is currently undetermined. In $\mathrm{CM}$, the organ distribution of sequestration is predominantly in the brain, although autopsy specimens show high levels of sequestration in other organs, such as the intestine [14]. In CM, end organ pathology is mainly in the brain, suggesting that this organ is uniquely unable to cope with the metabolic or inflammatory consequences of infection.

Sequestration has two broad consequences thought to lead to coma pathogenesis-mechanical obstruction of cerebral vasculature and initiation of an inflammatory response. The blockage of small diameter vasculature may be directly observed in vivo in the retinal veins of children with $\mathrm{CM}$, and in the mucous membranes of adults with CM $[15,20]$. Although there have been no studies directly observing cerebral blood vessels during life, post-mortem samples show high levels of sequestration in brain vasculature [12]. Vessel obstruction may be severe enough to result in hemostasis with an ensuing decrease in oxygen and nutrient delivery to the brain. Coupled with an increased metabolic demand brought on by fever and seizure activity, a significant metabolic mismatch can ensue, resulting in tissue hypoxia and neuronal dysfunction or death.

Sequestration of parasitized erythrocytes also initiates a powerful inflammatory cascade. At least two parasite products are thought to be important in the initiation of this response: glycosylphosphatidyl-inositol and hemozoin [21-24]. Both of these mediators stimulate the production of proinflammatory cytokines, including TNF- $\alpha$ and IL-1. These cytokines upregulate vascular endothelial receptors leading to enhanced sequestration. A positive feedback loop of increased sequestration and continued inflammation ensues.

Whether mediated by sequestration, inflammation or a combination of the two processes, there is evidence of blood-brain barrier (BBB) compromise (reviewed in [25]). In autopsy-derived samples from children with $\mathrm{CM}$, there are conflicting results with one group showing loss of tight junction proteins and a lack of transendothelial leakage of fibrinogen, whereas a more recent report shows significant leakage of fibrinogen into the brain parenchyma [26,27]. Assessment of the integrity of the BBB can be determined in vivo with the use of retinal fluorescein angiography. Leakage of dye signifies a disruption of the vascular barrier. In a study of 34 children with $\mathrm{CM}$, leakage occurred in $44 \%$ of subjects [15]. Despite the disruption of the BBB, there is a paucity of leukocytes in the brain parenchyma of autopsy samples; cellular inflammation is primarily intravascular. Disruption of the BBB and migration of intravascular fluid into cerebral parenchyma is probably important in the cerebral edema seen radiographically in some patients. In children who have died from CM, autopsies have shown cerebral ring hemorrhages thought to be the result of vascular compromise and microthrombus formation. Perturbation of endothelial cells by parasites may lead to a prothrombotic state, perhaps by induced expression of tissue factor [28].

Whatever the inciting perturbations, raised intracranial pressure and concomitant brain swelling may lead to cerebral herniation, the ultimate cause of death in many children with CM. Intracranial pressure, inferred from CSF opening pressure, was measured in a population of children with CM and found to be universally raised [29]. A subsequent study directly measuring intracranial pressure in 23 children with CM found all subjects had increased pressures, with mortality correlating with degree of intracranial hypertension. Brain swelling, whether as a result of a disruption of the BBB and vasogenic edema, or cytotoxic edema brought on by inflammation or hypoxia, has been well documented in CM. Autopsy studies show universally increased brain weight in children dying of $\mathrm{CM}$ [27], and CT and MRI studies show a large percentage of children with increased brain volume [30,31].

\section{Diagnosis}

The WHO defines CM as unrousable coma (Blantyre Coma Score of $\leq 2$ ) in the presence of any level of peripheral $P$. falciparum parasitemia, with no other discernible cause of illness (commonly, but not limited to, meningitis, hypoglycemia or postictal state). Across the developing world, clinicians may have varying 
access to technologies to diagnose these alternative coma etiologies. Postictal state may be ruled out across the spectrum of healthcare resource settings, but there is no clear duration of time in the WHO clinical definition. Therefore, if a child has fever, peripheral parasitemia and recovers consciousness in $30 \mathrm{~min}$ after a generalized seizure, centers may or may not diagnose CM. If serum glucose levels are not available, hypoglycemia as a coma contributor is often ruled out by empiric intravenous infusion of dextrose. Even if consent for lumbar puncture is given, laboratory facilities to process cerebral spinal fluid are often lacking in many settings. Consequently, empiric antibiotic treatment is often administered with antimalarials. In patients thus treated, clinical improvement cannot be attributed solely to either therapy, obscuring underlying disease diagnosis and epidemiology.

The poor specificity of the WHO clinical definition of $\mathrm{CM}$ is highlighted by considering community rates of asymptomatic parasitemia. Children with peripheral parasitemia, yet no clinical signs or symptoms of malaria, are widespread in areas of high malaria transmission [32]. Therefore, patients with an asymptomatic parasitemia and other underlying etiologies of coma (e.g., bacterial or viral infection, poisoning, intracerebral bleed or Reye syndrome) could easily be misclassified as having malaria as their sole coma etiology. These nonmalarial causes of coma may be the most common in malaria endemic regions, but are not exclusive. Trauma and overwhelming bacterial or viral infections may also lead to coma. In locations with high population rates of asymptomatic parasitemia, children with these nonmalaria coma contributors will meet the WHO clinical case definition for $\mathrm{CM}$ even though the parasitemia may not be the underlying etiology of their acute illness. The low specificity of the clinical diagnosis of CM is demonstrated by a large autopsy study of children dying of casedefined CM [12]. A total of $24 \%$ of children thus diagnosed did not have malaria parasite sequestration in cerebral vessels at autopsy. In all cases where sequestration was absent, children had alternative nonmalarial causes of coma identified including severe pneumonia and Reye syndrome. Differentiation of this sizable minority group during life was not possible until the discovery and description of malarial retinopathy.

A unique retinopathy in children with $\mathrm{CM}$ was first noted in 1877 by Mr Atkinson, a housephysician in London who noted hemorrhages in the retinas of six patients [33]. Not until 1996 was the full importance of this clinical finding made clear [34]. The presence of malarial retinopathy is $95 \%$ sensitive and $100 \%$ specific for the premorbid identification of cerebral parasite sequestration found at autopsy [35]. Malarial retinopathy may be seen on direct or indirect ophthalmoscopy and has three major findings: hemorrhages, whitening and vessel changes (orange or white discoloration). Papilledema may be present in addition to these three abnormalities, but is not diagnostic in isolation. The presence of malarial retinopathy confirms that the malaria parasite is responsible for the patient's acute illness and coma. Patients with clinically defined CM who are retinopathy negative are presumed to have various nonmalarial coma contributors. Owing to the need for personnel training to ascertain retinopathy status, its diagnosis may not be practical in all clinical settings. Development of a bedside blood test to differentiate children with malarial versus nonmalarial coma etiologies may be more practical in low-resource areas. Such testing is currently in development. In one case, a parasite-produced protein, HRP-2, is under testing as a CM predictive biomarker. HRP-2 levels have been shown to have an excellent ability to predict retinopathy status, with higher levels found in those who are retinopathy positive [36]. Further testing of this and other diagnostic modalities is ongoing.

\section{Treatment \\ Antimalarials}

Once a diagnosis of CM is entertained, treatment should begin immediately. Without treatment, the disease is universally fatal. The mainstays of therapy include supportive care, antimalarials and anticipation and treatment of complications. If available, patients should be managed in an intensive care unit or its local equivalent. Minimum requirements for standard care of CM patients in resource-poor contexts have been delineated (Box 1) [37].

Worldwide, the two most common classes of antimalarials used are cinchona alkaloids (quinine or quinidine) and derivatives of artemisinin (artesunate, artemether and others). Medication availability and national protocols may drive antimalarial selection. Both classes of antimalarials require a parenteral loading dose to rapidly achieve therapeutic drug levels.

Quinine and quinidine are alkaloids derived from the bark of the cinchona tree indigenous to the Andean mountains of South America. The bark has been used medicinally since the 
Box 1. Minimum requirements for standard care of children with cerebral malaria in resource-limited settings.

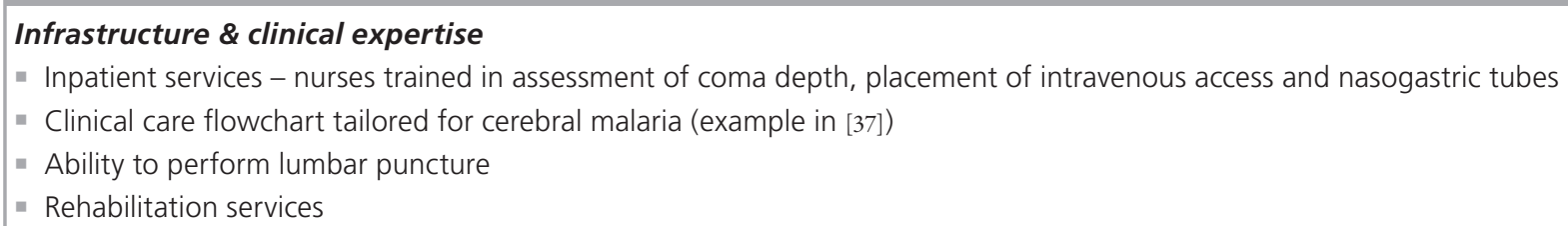

Laboratory

- Rapid testing for glucose and intravenous glucose for administration

- Malaria diagnostics - thick smear for parasites and/or rapid diagnostic tests

- Gram stain for cerebral spinal fluid

\section{Medications}

- Intravenous antimalarials - artesunate or quinine

- Anticonvulsants - intravenous preparations preferred

- Broad spectrum intravenous antibiotics

- Enteral nutrition preparations

Data taken from [37].

16th century, and was introduced as a fever cure in Europe as early as the 17 th century. These alkaloids act only on later stages of parasite development and are still used in many countries as the antimalarial of choice or necessity. Only quinine is available orally, but both quinine and quinidine can be administered parenterally. Side effects of either agent are numerous and common. They include hypoglycemia, as both increase endogenous insulin secretion. The side effect syndrome termed 'cinchonism', although rare, includes tinnitus, high-frequency hearing loss and color visual aberration [7]. Quinidine may induce cardiac dysrhythmias. If available, patients should have continuous cardiac monitoring during its infusion.

Artemisinin and its related pharmacologically active compounds (artesunate, artemether and dihydroartesunate) are derivatives of wormwood, used for centuries in China as therapy for fever. In the 1970 s, spurred by continued malarial deaths in north Vietnam, a massive scientific effort by the Chinese government identified and purified artemisinin as the active compound in this traditional remedy [38]. These medications kill parasites in all stages of the asexual and sexual life cycle. With activity during a wider spectrum of the parasite life cycle, artemisinin derivatives clear parasites more rapidly than cinchona alkaloids. There are fewer side effects of artemisinin derivatives compared with quinine and quinidine [39].

A meta-analysis of randomized controlled trials comparing arteminisin derivatives and cinchona alkaloids in the treatment of pediatric $\mathrm{CM}$ showed no differences in mortality or neurologic outcomes [39]. A subsequent multisite open-label study of artesunate versus quinine in over 5400 African children with severe malaria (including but not confined to $\mathrm{CM}$ ) showed a significant decrease in mortality in those treated with artesunate (odds ratio: -0.75; 95\% CI: -0.63 to -0.90 ) [40]. A subgroup analysis of those with CM revealed nonstatistically significantly different mortality rates of $21 \%$ in those treated with quinine versus $18 \%$ in the artesunate arm. Rates of neurologic morbidity in survivors were not affected by antimalarial choice, although the proportion of survivors with neurologic sequelae in this study was much lower than found in more recently published cohort studies of cerebral malaria survivors [10,11]. Artemisinin derivatives are now the treatment of choice for all forms of malaria and are not inferior to cinchona alkaloids for the treatment of CM. Artesunate is available in the UK, but not in the EU. Until recently, quinidine was the only parenteral treatment for CM available in the USA. In mid-2010, intravenous artesunate was US FDA approved and is now available through the CDC [41].

Unfortunately, when used as monotherapy or in subtherapeutic doses, artemisinin monotherapy may induce the emergence of drug resistance [42]. Although clinical resistance has not yet been seen, delayed parasite clearance has been observed in malaria cases along the Thai-Cambodia border [43]. This possible step along the path to resistance has led to the recommendation that short half-life arteminisin derivatives should be combined with a longer acting partner drug. The most common combinations are with mefloquine, amodiaquine, piperaquine or lumefantrine. These arteminisinin 
combination therapies are now the treatment of choice for all forms of malaria [44]. This policy has been endorsed by the WHO.

\section{Supportive care}

In addition to appropriate antimalarial medication, children with CM require assiduous monitoring to detect and prevent any complications that may lead to poor outcome. In many patients, the disease has a dramatically rapid onset and resolution. Although antimalarials clear parasites rapidly, vigilance to other clinical parameters during this time may improve the chance of full recovery. Specific attention should be given to hydration and detection and treatment of hypoglycemia, anemia and seizures. Treatment of coinfections with bacterial pathogens and optimizing nutritional state are extremely important in clinical care.

\section{Parenteral fluids}

Fluid administration in malaria is controversial as the hemodilution from intravenous infusion may further exacerbate the anemia that results from erythrocyte sequestration and lysis. Metabolic acidosis is common in CM and may be caused by increased production and decreased metabolism of tissue acids, anemia, hypovolemia and fever $[45,46]$. The choice of intravenous fluid most appropriate for volume expansion in children with CM is controversial. Although various studies have shown superiority of colloid over crystalloid, a recent meta-analysis found no difference in clinical recovery time from septic shock (including children with malaria) regardless of the fluid used [47]. A subanalysis of the trials limited to malaria revealed a slight increase in the rate of survival in children receiving colloids compared with crystalloids. In low-resource settings, choice of parenteral fluid may be dictated by availability and cost.

A recent multicenter trial in Africa examined mortality after intravenous fluid bolus in children with severe infection. In total, $57 \%$ of patients had malaria [48]. Children who received fluid boluses had higher rates of mortality and neurologic morbidity than those who received maintenance intravenous fluids only. These results do not support routine use of bolus intravenous fluid resuscitation in ill children with impaired perfusion. It is possible that rapidly reversing the body's compensatory mechanisms to severe infection and shock is detrimental.

In summary, unless a child is clinically at risk of imminent death from circulatory collapse, CM patients should be administered intravenous fluids at maintenance rates. Due to the risk of hypoglycemia from the underlying disease (further exacerbated in children treated with cinchona alkaloids; see section entitled 'Treatment'), fluids should contain dextrose. Commonly used choices include half-strength Darrow's with $5 \%$ dextrose or $0.45 \%$ normal saline with 5\% dextrose. When the patient is able to take fluids or feeds enterally, intravenous fluids can be stopped.

Identification \& treatment of hypoglycemia Several etiologies have been described for CM-related hypoglycemia. They include acute lack of food intake, impairment of hepatic gluconeogenesis, parasite consumption of glucose, hyperinsulinism induced by cinchona alkaloids and increased metabolic demands of the host [49]. Hypoglycemia is a known independent risk factor for death $[9,49]$ in children with severe malaria and is present in $15 \%$ of children. A total of $70 \%$ of hypoglycemic episodes occur within $24 \mathrm{~h}$ of hospital admission [50]. Hypoglycemia may not be clinically apparent in patients with $\mathrm{CM}$ and it is imperative to monitor serum glucose to assure early recognition and correction [50,51]. When hypoglycemia is detected, it should be corrected with $5 \mathrm{ml}$ per kilogram of intravenous $10 \%$ dextrose, if available. A glucose level should be rechecked after 15-20 min to ensure adequate correction.

A randomized controlled trial compared the efficacy of sublingual sugar and intravenous glucose for treatment of hypoglycemia [52]. The primary outcome was correction of hypoglycemia within $40 \mathrm{~min}$ of diagnosis. Sublingual sugar was equally effective as intravenous glucose in the initial correction of hypoglycemia. However, relapse of hypoglycemia was more frequent in patients randomized to sublingual sugar. In situations where intravenous administration of glucose is not feasible or available, sublingual administration of sugar may be considered for temporary correction of hypoglycemia.

\section{Identification \& treatment of anemia}

Anemia is a major cause of morbidity and mortality in sub-Saharan Africa, particularly among children in areas of high malaria transmission [53]. A majority of these children already have mild-to-moderate anemia, predisposing them to severe anemia during acute malaria. In children, the case fatality rate of complicated malaria increases with young age and transmission intensity [54]. 
Children with severe anemia have a high risk of mortality that extends beyond the period of hospitalization, and are therefore often transfused [54-56]. In children with nonsevere anemia, the decision to transfuse becomes less straightforward. Although transfusion may shorten hospital stay and lower the risk of mortality, it has potential risks including circulatory overload, transfusion reactions and infection with HIV and hepatitis [54,55]. A Cochrane review evaluated routine blood transfusion for those with hematocrits between 12 and $17 \%$ [57]. This review encompassed two randomized controlled trials including a total of 230 children with malaria-associated severe anemia. There was no significant difference in the risk of death or adverse outcome between children who were transfused and those who were not (relative risk: 2.54, 95\% CI: 0.69-9.34). The WHO recommends that red blood cell transfusion be administered only if anemia is associated with incipient or established cardiac failure [58].

\section{Prevalence, identification \& treatment of seizures}

Seizures are common in children with severe malaria. Up to $60 \%$ of children with malaria will experience seizures in the course of their illness $[59,60]$. Prolonged and repetitive seizures are associated with death and neurological sequelae in children with CM and are a risk factor for the development of epilepsy [10,60]. Seizures may be clinically evident or nonconvulsive, identifiable only by epileptiform activity on EEG. The prevalence of subclinical seizures was clarified in a recent cohort study of children with retinopathy-positive CM where $19 \%$ of children who had no witnessed seizures prior to or during admission had epileptiform activity on EEG [10].

Seizure treatment algorithms often begin with benzodiazepines, most commonly diazepam or lorazepam. Both are rapidly acting, inexpensive and may be administered intravenously or rectally. Diazepam administered intravenously achieves therapeutic levels within $5 \mathrm{~min}$. Although there is significant pharmacokinetic variability with rectal administration, this route is crucial in lowresource settings where intravenous access is often not feasible [60]. Benzodiazepines are associated with cardio-respiratory depression (especially when combined with barbiturates such as phenobarbitone) and repetitive administration or continuous infusions are inadvisable without inotropic or ventilator support. For patients with acute seizures refractory to benzodiazepines or in whom a maintenance anticonvulsant is required, phenobarbitone or phenytoin are most commonly used in resource-limited settings.

\section{Antibiotics}

Empiric use of antibiotics in children with CM remains controversial. The presence of malarial parasitemia does not exclude concurrent bacteremia. Acute hemolysis during malaria may predispose children to bacteremia. Free heme, produced when the malaria parasite lyses an erythrocyte, leads to upregulation of heme oxygenase. This enzyme impairs the oxidative burst in granulocytes. This impairment provides an advantage for certain intracellular bacteria, including nontyphoidal Salmonella [61,62].

In a recently published cohort study, over $4 \%$ of Malawian children with severe malaria had concurrent bacteremia [63]. Children who were younger, had more severe anemia, and those with a high white blood cell count were at greater risk of bacterial coinfection. HIV infection was not a clear risk factor for bacteremia in this population. Use of empiric antibiotic therapy did not improve outcome [63].

Salmonella is the most common bacterial coinfection in children with severe malaria [63-65]. Current WHO treatment guidelines suggest a low threshold for beginning antibiotic therapy in these children. Patients in whom antibiotics should be strongly considered include those who are severely anemic and have deep breathing, are malnourished or exhibit signs of shock $[37,65,66]$. A child that clears parasites and remains febrile may warrant commencement of antibiotics. Consideration must be given to the possibility that sepsis is the primary cause of coma with malaria parasitemia being asymptomatic, especially in children without malarial retinopathy (see section entitled 'Diagnosis').

\section{Nutritional support}

Although the disease course of CM may be short, nutritional support is often warranted [67]. A recent clinical trial of children and adults with CM compared the effect of early and late commencement of enteral feeds in a setting where intubation was not available [68]. Early enteral feeding was started on the day of admission, while late enteral feeding was begun after $60 \mathrm{~h}$ in adults or $36 \mathrm{~h}$ in children [68]. The trial was stopped after 56 subjects were enrolled owing to a high rate of aspiration pneumonia in the early feeding group. Four of the 56 subjects were children and none of the aspiration events 
occurred in these four. Extension of these findings to the entire pediatric CM population is problematic and focused evaluation of the pediatric population is warranted. The timing of commencement of enteral feeding may depend on nursing and clinician supervision. If adequate staffing is available, close monitoring of early nasogastric feeds can identify patients not tolerating feeds well. In this case, nutrition by the enteral route can be deferred and full volume intravenous fluids reinstated.

\section{Adjunctive therapy}

The need for adjunctive therapy for CM is highlighted by a high mortality rate despite prompt treatment with antimalarial therapy [69]. Trials with various adjunctive therapies have been performed over the years and none have shown a decrease in mortality. Some trials have resulted in increased mortality in the treatment over the control arm. A select group of completed trials is highlighted below. A more comprehensive list of trials may be found in Enwere [70].

\section{Anticonvulsants}

Children with CM often have seizures that are difficult to control. Anticonvulsants are not routinely administered to children who do not have clinical or electrographic (where EEG is available) evidence of seizures. Believing that seizures are central to disease pathogenesis and not an epiphenomenon, Crawley et al. randomized 340 children (regardless of clinical seizure activity) to either a single dose of adjunctive phenobarbital $(20 \mathrm{mg} / \mathrm{kg})$ or placebo [71]. Although children randomized to the phenobarbital treatment arm experienced significantly fewer seizures, their mortality rate was more than double that of the children treated with standard care alone. Increased mortality was attributed to phenobarbital-induced respiratory depression. Survivors in the adjunctive phenobarbital group experienced fewer neurological sequelae than those randomized to placebo. This difference was interpreted with caution as differences in survival rates may mask sequelae. Clinical trials of anticonvulsants with lower rates of respiratory depression are warranted.

\section{Corticosteroids}

Before 1982, corticosteroids were commonly used as adjunctive therapy for CM with presumed cerebral edema as their target. In a clinical trial from 1982, 100 Thai CM patients of all ages were randomized to either dexamethasone or placebo [72]. No difference in survival rates was seen. Subjects randomized to adjunctive corticosteroids had significantly higher rates of pneumonia and gastrointestinal bleeding. A subanalysis by age demonstrated identical survival rates between treatment arms in pediatric patients.

A second double-blind placebo-controlled trial of adjunctive corticosteroids in children and adults and conducted in southeast Asia used a dexamethasone dose more than five-times higher [73]. Dosage was chosen based on previous success in the treatment of typhoid fever [74]. Thirty eight subjects were randomized and there was no difference in survival outcome. Secondary outcomes of fever clearance, time to return to consciousness and parasite clearance time did not differ between treatment groups. A subanalysis by age was not possible given the small number of subjects.

\section{Anti-TNF- $\alpha$}

Inflammation with subsequent activation of endothelial cells is hypothesized to be important in disease pathogenesis (for a review, see [75]). TNF- $\alpha$ levels have been correlated with malaria disease severity and antibody neutralization of TNF- $\alpha$ can reverse disease in murine models of CM [76-78]. Based on these findings, antiTNF- $\alpha$ antibody therapy was hypothesized to be possibly effective in human disease. In a randomized controlled trial, 610 children with CM between 1 and 9 years old were randomized to standard CM care and either anti-TNF- $\alpha$ antibody or placebo [79]. Primary outcomes were mortality and neurological sequelae at 6 months posthospital discharge. Although no difference was seen in mortality rates, significantly more children in the anti-TNF- $\alpha$ group had neurological sequelae at 6 months compared with those given placebo (6.8 vs $2.2 \% ; \mathrm{p}=0.02)$.

\section{Inhaled nitric oxide}

Recent data implicates endothelial cell activation as a possible contributor to $\mathrm{CM}$ pathogenesis (see section entitled 'Pathogenesis'). Specific markers of endothelial cell activation, such as Ang-2, have been associated with poor outcome [80]. Production of nitric oxide by endothelial cells has multiple effects, including vasorelaxation, inhibition of platelet aggregation and downregulation of endothelial adhesion molecules, all of which could lead to protection against CM. The short half-life of nitric oxide, as well as its ability to diffuse quickly makes direct measurement difficult. However, decreased 
levels of the precursor to nitric oxide, E-arginine, have been shown to correlate with malaria disease severity in children [81]. Based on these data, as well as encouraging results showing that increased nitric oxide may lead to disease protection in a mouse model of CM [82], there are two ongoing randomized controlled trials of inhaled nitric oxide as adjunctive treatment of CM taking place in Uganda [83,84].

There may be several reasons for the failure of previous adjunctive therapy trials to demonstrate lower mortality rates in the treatment arm. First, the target of the adjunctive therapy may only be peripherally involved in the disease process. Its neutralization may not alter more important determinants of pathophysiology. Second, many adjunctive therapy trials have been underpowered. Previous studies have used the WHO clinical definition of $\mathrm{CM}$ as an inclusion criterion. This definition is nonspecific. An autopsy study showed that $24 \%$ of children dying of CM lacked cerebral parasite sequestration and had other nonmalarial explanations for coma [12]. Inclusion of this group within the entire study population may have led to nondifferential misclassification with resultant loss of study power. Stratification by retinopathy status (see section entitled 'Pathophysiology') may improve power in future adjunctive therapy trials. Third, symptom progression in $\mathrm{CM}$ is often rapid, going from asymptomatic to coma or death in less than $48 \mathrm{~h}$. It is possible that by the time children present to medical care, the disease is too far advanced for adjunctive therapy to be effective.

If this third possibility is accurate, efforts should instead be intensified on early treatment and prevention. A longitudinal study of three alternate antimalarial treatments for uncomplicated malaria in Uganda has provided insight into the effect of rapid malaria treatment [85]. A total of 690 children were followed for up to 4 years. The cohort was administered one of three antimalarial regimens upon any sign or symptom of illness compatible with clinical malaria. Insecticide-treated bednets were distributed. Over the 4-year follow-up period more than 1400 episodes of uncomplicated malaria were detected. There were no cases of severe or $\mathrm{CM}$ in any of the treatment arms. Although there was no control group to document $\mathrm{CM}$ incidence in the absence of intervention, a standard CM rate of $1 \%$ of total malaria cases suggests that at least some cases should have been seen. Rapid effective treatment of uncomplicated malaria, along with preventive methods, such as bednet distribution, may play an important role in preventing the progression of uncomplicated disease to CM. Efforts focused on prevention and rapid effective treatment may be complementary to investigations into effective adjunctive therapies.

\section{Outcomes \\ Neurosequelae}

In addition to the mortality associated with $\mathrm{CM}$, there is an overwhelming burden of morbidity associated with survivors. Major neurologic deficits, including epilepsy, neurodevelopmental disorders and cognitive-behavioral problems are seen in 30\% of those who survive their acute illness $[10,11]$. Other studies estimate rates of cognitive deficits alone as 24 and $29 \%$. These deficits are manifested in the areas of attention, memory, tactile-based perception and language [86].

Why some children recover from CM with no neurological consequences, while others are severely debilitated may be partially explained by the heterogeneous nature of coma in patients with clinically defined CM. Although onequarter of children given this clinical diagnosis probably have a nonmalarial cause of coma (and are retinopathy negative, see 'Diagnosis' section), the odds of adverse neurologic outcome in CM survivors is the same whether malarial retinopathy is present [10] or not [11]. Children may be diagnosed with $\mathrm{CM}$ in the setting of uncomplicated malaria and a prolonged postictal state. These children would be unlikely to suffer the same long-term neurodisabilities as those with systemic and cerebral pathologies leading to prolonged coma.

\section{Neuroanatomical basis of $\mathrm{CM}$ cognitive sequelae}

Brain MRI scans in children with CM reveal periventricular white matter lesions as one of the most common abnormalities [31]. The corpus callosum and forebrain commissures, critical in interhemispheric communication and processing, may be damaged due to axonal demyelination and local cerebral hypoxic effects [87,88]. Focal cognitive deficits documented in $\mathrm{CM}$ may be the consequence of injury to interand intra-hemispheric connective pathways with temporal lobe/hippocampal regions particularly vulnerable to neurovascular compromise and subsequent hypoxic/ischemic effects.

EEG studies of children during the acute illness and follow-up reveal posterior parietotemporal discharges and diffuse slow-wave abnormality, especially in those with persisting 
neurological sequelae [89]. MRI of children with seizures during their acute illness who have subsequent neurodisabilities reveals focal cortical atrophy, diffuse cerebral atrophy, isolated vermian atrophy and communicating hydrocephalus [10,31].

Attention-deficit/hyperactivity disorder (ADHD)-like symptoms have been described in CM survivors [10]. Idro and colleagues observed significant behavior problems in 11 of 23 surviving Ugandan CM children at a neurodisability clinic. They characterized these behavior problems as consisting of "hyperactivity, impulsiveness and inattentiveness as in ADHD and conduct disorders with aggressive, self injurious or destructive behavior" [90]. Twelve of 21 Malawian CM surviving children referred for behavioral problems were diagnosed with DSM IV axis $1 \mathrm{ADHD}$, and 15 had a diagnosis of oppositional defiant disorder [91].

We should caution that behavioral problems are common after other brain injuries and are not specific to CM. However, from a brain/ behavior standpoint, the prevalence of ADHDtype symptoms is significant in light of the abnormalities in diffusion-weighted MRI in the white matter of the centrum semiovale implicated in case studies of children with CM [92]. MRI spectroscopy assessments of 22 Spanish non-CM patients with ADHD-like symptoms revealed increased $N$-acetylaspartate/ creatine ratios in the right prefrontal subcortical region and left centrum semiovale, compared with eight healthy controls [93]. No structural differences were apparent. $N$-acetylaspartate and creatinine kinase can both be readily detected in the human brain with MRI spectroscopy. The ratio of these two substances provides a sensitive biomarker of possible brain injury and atrophy in neuronal networks. A high ratio of this brain injury biomarker in white matter regions of the brain is implicated in children with CM who have behavioral sequelae, as well as in non-CM ADHD children and suggests perhaps a common brain/behavior etiology [92,93].

It has been hypothesized that in young children with CM, microvascular sequestration, mitochondrial hypermetabolism, hemorrhage and $\mathrm{BBB}$ disruption can compromise arterial watershed regions serving the white matter and other major connective pathways of the brain [94]. Recent functional MRI studies of medication-naive children with ADHD have revealed inferior frontal and temporalparietal brain region dysfunctions related to learning, memory, attention and impulsivity problems [95-97]. Neural networks in brain regions mediating these executive processes are especially vulnerable to hypoglycemic coma, BBB compromise and hypoxic-ischemic crisis in children [98-100]. All of these events can play a role in the brain pathology following CM [90]. When these regions are metabolically injured, the behavioral impact can be expressed by ADHD-like symptoms.

\section{Cerebral spinal fluid markers of $\mathrm{CM}$ cognitive sequelae}

John and colleagues evaluated cerebral spinal fluid (CSF) and serum levels of 12 cytokines or chemokines important in CNS infections in 76 Ugandan children hospitalized with CM and eight control children [101]. Elevated CSF TNF- $\alpha$ levels on admission were associated with an increased risk of neurologic deficits 3 months later (odds ratio: 1.55, 95\% CI: 1.10 $2.18 ; \mathrm{p}<0.01)$. TNF- $\alpha$ was also negatively correlated with age-adjusted scores for the Test of Variables of Attention (Spearman rho: -0.34; $\mathrm{p}<0.04)$ and the Kaufman Assessment Battery for Children working memory (Spearman rho: $-0.32 ; p<0.06) 6$ months later. This study was the first to document that CSF TNF- $\alpha$ production is associated with neurologic and cognitive sequelae. The mechanisms by which the excessive TNF- $\alpha$ in the brain can lead to neurological injury is well documented elsewhere [102].

In a subsequent computerized cognitive rehabilitation training study with this same cohort of children approximately 3 years after their illness [103], TNF- $\alpha$ levels during the acute illness was significantly negatively correlated with neuropsychological memory and learning performance prior to cognitive rehabilitation training intervention. Statistically significant negative Spearman correlation coefficients were obtained between CSF TNF- $\alpha$ during illness and computerized maze learning $(\mathrm{p}=0.03)$, card detection (attention; $\mathrm{p}=0.025$ ), card identification (two-step attention; $p=0.009$ ) and card one-back working memory task $(\mathrm{p}=0.02)$ immediately prior to cognitive rehabilitation training intervention. CSF TNF- $\alpha$ was also significantly correlated with neuropsychological improvements on a computerized maze visualmotor tracking task from before compared to after training $(p=0.015)$ [Borvin M, Pers. Сomm.].

\section{Neuropsychological sequelae of CM}

The first documented neuropsychological deficits in pediatric CM survivors showed deficits 
on visual scanning (a measure of attention), immediate and delayed visual memory, bimanual tactile discrimination, perceptual abstraction and rule learning skills, right ear auditory information processing and dominant hand motor speed. Deficits were related to coma duration [87,88].

Subsequent studies with CM survivors demonstrated poor performance on the Kaufman Assessment Battery for Children sequential processing subscales of Hand Movements and Word Order and on the simultaneous processing scale of Spatial Memory compared with healthy controls [104]. CM survivors also did more poorly on the principal measure of attention on the Test of Variables of Attention [104].

Attention and working memory deficits are, therefore, important in assessing the long-term neurocognitive sequelae of CM. CM survivors have a 4.6-fold increased risk of a cognitive deficit as compared with unexposed controls after adjustment for age, gender, nutritional status, school level and home environment. Among children with CM, increased number of seizures prior to admission and longer coma duration during hospitalization are predictors for cognitive deficits at 6-month follow-up [86]. Other studies have demonstrated that children with a history of recurring seizures during acute illness are at risk for cognitive and language impairment [51,105-108]. Attention, memory, learning and language impairment from CM can place children at a significant disadvantage in such functional domains as academic achievement and day-to-day adaptive behaviors related to executive function at home or in the community [109-112].

The long-term effects of malarial illness may extend beyond attention and memory ability in the immediate aftermath of acute illness $[10,112]$. Recent findings suggest that such effects could accrue incrementally with repeated bouts of uncomplicated malaria [113]. In one clinical trial, intermittent preventative treatment (IPTc) of malaria in Kenyan school children significantly enhanced two of the class-based tests of sustained attention [114]. The authors concluded that IPTc of malaria improves the health and cognitive ability of semi-immune school children.

\section{Cognitive rehabilitation in children surviving $\mathrm{CM}$}

There is no known treatment intervention during acute illness that decreases rates of cognitive sequelae in CM survivors [15]. Neurocognitive rehabilitative treatment programs are usually unavailable in the low-resource settings where most affected children live. A computerized cognitive rehabilitation therapy has recently been successfully piloted in CM survivors [103]. A randomized controlled trial of this therapy targeting pediatric severe malaria survivors is in process with end points targeting abnormalities in working memory, executive functioning and psychosocial adjustment.

\section{Developmental enhancement for $\mathrm{CM}$ preschool-age survivors}

Interventions to train caregivers of younger CM survivors in ways that will enhance the development of their children in day-to-day interactions in the home are needed. Such interventions are available in a wide range of cultural settings and across varied populations of children with special needs. Training may improve caregiver-child interactions in a way that promotes development and growth even in the face of adversity. Caregiver training to enhance the development of at-risk children in rural low-resource settings has been effective in HIV-positive children [116]. Trials of similar interventions in children suffering from post-CM sequelae are warranted.

\section{Prevention strategies}

A three-part strategy of malaria eradication is now widely endorsed [117]. This consists of: aggressive control of mosquitoes in areas of high malaria endemicity; elimination of malaria from the margins of endemic areas, moving progressively towards level of higher malaria transmission; and improved primary prevention, diagnostic and treatment strategies.

Primary prevention strategies are currently focused in three main areas: bednets, chemoprophylaxis and vaccine development. Insecticide-treated bednets are the most widely used strategy for those living in malaria endemic areas. Anopheles mosquitoes, the only vector capable of transmitting Plasmodium, usually bite outside and at night. Remaining indoors or under a bednet at these times decreases malaria risk. Insecticide-impregnated bednets are superior to unimpregnated nets. Even with intermittent washing, impregnated nets provide protection for approximately 1 year [118]. The use of impregnated bed nets lowers pediatric population all-cause mortality rates by up to $60 \%[119]$.

Chemoprophylaxis is often prescribed to temporary visitors to malaria endemic regions. In young (1-4 years old) residents of endemic areas with high transmission intensities, prophylaxis 
has been shown to decrease mortality rates by $25 \%$ [120]. Owing to concerns about possible encouragement of the emergence of drugresistant parasite strains with prolonged use of prophylaxis, this public health approach has not been widely implemented.

Intermittent preventive therapy is a strategy where antimalarial therapy is given at regular intervals independent of the presence of parasitemia. Intermittent preventive therapy in infants (IPTi) usually involves doses of sulphadoxine-pyramethamine (SP) at 2, 3 and 6 months of age. These doses are combined with regularly scheduled vaccines. In a meta-analysis of six trials of IPTi using SP, the intervention decreased malaria episodes by $30 \%$, anemia by $21 \%$, malaria hospital admissions by $38 \%$ and overall hospital admissions by $23 \%$ [121]. However, a randomized clinical trial of IPTi using mortality as an outcome showed no benefit [122]. The WHO conditionally recommends IPTi for children in moderate-tohigh transmission areas where resistance to SP is less than $50 \%$ [123]. Intermittent preventive therapy can also be extended beyond infancy to children (IPTc). IPTc regimens vary in dosage as well as choice of antimalarial. One commonly used regimen involves oral antimalarials every month or every other month for the duration of the malaria transmission season. In a metaanalysis combining seven randomized controlled trials of this strategy, IPTc prevented $74 \%$ of clinical malaria episodes [124]. Although there was a trend toward a decrease in mortality among the group receiving IPTc, study power was insufficient to test statistical significance. At this time, the WHO does not recommend IPTc for any location except the Sahel subregion of Africa. In this area it is recommended that children aged 3-59 months old receive monthly doses (maximum of four) of amodiaquine plus SP during the highly seasonal malaria transmission season. IPTc has recently been renamed seasonal malaria chemoprevention (SMC). There is no current WHO recommendation for SMC in other geographic contexts. Drug resistance is a concern, but a further impediment lies in the lack of a structured setting for delivery since medications would be administered outside the standard vaccine schedule.

If malaria eradication is to be achieved, an effective vaccine will probably be one of its most important strategies. Proof-of-principle for vaccine efficacy occurred more than 30 years ago when the bite of multiple irradiated mosquitoes resulted in effective antimalarial protection [125].
Despite intense efforts, a universally effective antimalaria vaccine is not yet available. There are multiple pathogen-specific barriers to effective vaccine development. Perhaps the greatest of these is the complexity of the malaria parasite with its more than 5000 genes and a complicated and incompletely understood gene regulation. Antigenic targets are therefore not obvious. Multiple genotypes would probably need to be included in an effective vaccine. Choice of immunogens is also hampered by lack of a defined immune response that clearly correlates with protection from disease. In the absence of such a marker of immune protection, rational immunogen choice is hampered.

Despite these challenges, there are over 25 distinct antigens and vaccine formulations that have progressed to Phase I evaluation [126]. The candidate furthest along the developmental pathway is RTS, S/AS01. The antigenic target is CSP expressed on sporozoites. Phase II trials showed a 38\% efficacy against severe disease. A large multicentered Phase III trial is underway and early results show a 50\% reduction in clinical malaria episodes in the vaccinated group [127]. Although these levels of protection would not be acceptable for many other pathogens, this is a significant accomplishment and could serve as one element in a multipronged malaria control approach. Other possible avenues for improvement in vaccine efficacy include a multiantigen vaccine, prime/boost delivery and exploration of new adjuvants (for a review, see [128]).

Use of whole organisms as vaccine antigens was not a viable option until recently. This was owing mainly to the great difficulty required to aseptically isolate sufficient numbers of sporozoites. Continued incremental increases in sporozoite production and isolation techniques, along with a large-scale investment of effort to this goal have overcome many of these barriers, and large numbers of sporozoites are now being isolated. Recent trials in animal models with inactivated sporozoites resulted in suboptimal immune responses when the antigen was delivered intradermally [129]. Investigation of other delivery routes suggest that the method of delivery may be important, and intravenous delivery led to significantly better immune responses than the subcutaneous route. Clinical trials using intravenous delivery of inactivated sporozoites are warranted.

\section{Conclusion \& future perspective}

Continuing falls in malaria transmission intensity in sub-Saharan Africa will change the 
epidemiology of cerebral malaria. Although the disease is now essentially limited to young children, as transmission falls a higher proportion of those who fall ill will probably be older children, adolescents and adults. This may change disease presentation and prognosis. In the past 20 years, the underlying pathophysiology of the disease has been intensively investigated. Adjunctive therapy trials targeting the most important of these pathological processes will continue, hopefully with designs of adequate study power to ascertain treatment effects. Physical and cognitive rehabilitation of survivors is in its infancy, but resources for such care are becoming increasingly available. The development of preventive strategies, especially the wide distribution of bednets and an effective malaria vaccine may be the most important strategy in limiting this scourge of African children.

\section{Acknowledgments \\ The authors thank H Ackerman and the librarians at Countway library for assistance with historical references.}

\section{Executive summary}

\section{Epidemiology}

- The incidence of malaria is decreasing in some parts of sub-Saharan Africa, although the disease remains the most important parasitic disease of mankind.

\section{Pathogenesis}

- The most important pathophysiologic process of cerebral malaria is probably erythrocyte sequestration. Infected erythrocytes express parasite-encoded proteins that lead to irreversible binding to vascular endothelium. In cerebral malaria (CM), sequestration of erythrocytes to cerebral vasculature is intense.

- Other pathophysiologic processes of importance include inflammation and breakdown of the blood-brain barrier.

\section{Diagnosis}

- The WHO's clinical definition of CM has low specificity and includes children with nonmalarial causes of coma coupled with asymptomatic parasitemia.

- The presence or absence of a malarial retinopathy may differentiate patients who have malaria as their primary coma etiology, from those who have nonmalarial causes of coma.

\section{Therapy}

- Artemisinin derivatives are recommended by the WHO as treatment for all forms of malaria, including CM.

- In addition to antimalarials, supportive care of children with CM is extremely important. This includes detection and treatment of hypoglycemia, seizures and anemia.

- Phenobarbital, corticosteroids and anti-TNF- $\alpha$ antibodies have all been studied as adjunctive therapy in CM and none have been shown to improve outcomes.

- Adjunctive therapy trials have been limited by low study power and nondifferential misclassification.

\section{Outcomes}

- The mortality rate of CM is $15-25 \%$ and $30 \%$ of survivors have major neurologic sequelae.

- Cerebral malaria is a risk factor for the development of epilepsy, neurodisabilities, a disruptive behavioral disorder and cognitive impairment.

\section{Prevention strategies}

- Bednets, chemoprophylaxis and vaccines will all be important in malaria control and elimination programs.

- Malaria vaccines that provide partial protection from uncomplicated and severe illness are currently in Phase III efficacy trials.

\section{References}

1. WHO. WHO World Malaria Report. WHO, Geneva, Switzerland, 2010.

2. Lacerda MV, Fragoso SC, Alecrim MG et al. Postmortem characterization of patients with clinical diagnosis of Plasmodium vivax malaria: to what extent does this parasite kill? Clin. Infect. Dis. 55(8), e67-e74 (2012).

3. Mahgoub H, Gasim GI, Musa IR, Adam I. Severe Plasmodium vivax malaria among Sudanese children at New Halfa Hospital, Eastern Sudan. Parasit. Vectors 5(1), 154 (2012).
4. Parakh A, Agarwal N, Aggarwal A, Aneja A. Plasmodium vivax malaria in children: uncommon manifestations. Ann. Trop. Paediatr. 29(4), 253-256 (2009).

5. Tanwar GS, Khatri PC, Sengar GS et al. Clinical profiles of 13 children with Plasmodium vivax cerebral malaria. Ann. Trop. Paediatr. 31(4), 351-356 (2011).

6. Antinori S, Milazzo L, Ridolfo AL, Galimberti L, Corbellino M. Severe Plasmodium vivax malaria: fact or fiction? Clin. Infect. Dis. 55(11), 1581-1583 (2012).

7. Idro R, Ndiritu M, Ogutu B et al. Burden, features, and outcome of neurological involvement in acute falciparum malaria in Kenyan children. JAMA 297(20), 2232-2240 (2007).

8. Carme B, Bouquety JC, Plassart H. Mortality and sequelae due to cerebral malaria in African children in Brazzaville, Congo. Am. J. Trop. Med. Hyg. 48(2), 216-221 (1993).

9. Molyneux ME, Taylor TE, Wirima JJ, Borgstein A. Clinical features and prognostic indicators in paediatric cerebral malaria: a study of 131 comatose Malawian children. Q. J. Med. 71(265), 441-459 (1989).

10. Birbeck GL, Molyneux ME, Kaplan PW et al. Blantyre Malaria Project Epilepsy Study 
(BMPES) of neurological outcomes in retinopathy-positive paediatric cerebral malaria survivors: a prospective cohort study. Lancet Neurol. 9(12), 1173-1181 (2010).

11. Postels DG, Taylor TE, Molyneux M et al. Neurologic outcomes in retinopathy-negative cerebral malaria survivors. Neurology 79(12), 1268-1272 (2012).

12. Taylor T, Fu W, Carr R et al. Differentiating the pathologies of cerebral malaria by postmortem parasite counts. Nat. Med. 10(2), 143-145 (2004).

13. MacPherson GG, Warrell MJ, White NJ, Looareesuwan S, Warrell DA. Human cerebral malaria. A quantitative ultrastructural analysis of parasitized erythrocyte sequestration. Am. J. Pathol. 119(3), 385-401 (1985).

14. Seydel KB, Milner DA Jr, Kamiza SB, Molyneux ME, Taylor TE. The distribution and intensity of parasite sequestration in comatose Malawian children. J. Infect. Dis. 194(2), 208-205 (2006).

15. Beare NA, Harding SP, Taylor TE, Lewallen S, Molyneux ME. Perfusion abnormalities in children with cerebral malaria and malarial retinopathy. J. Infect. Dis. 199(2), 263-271 (2009).

16. Avril M, Tripathi AK, Brazier AJ et al. A restricted subset of var genes mediates adherence of Plasmodium falciparum-infected erythrocytes to brain endothelial cells. Proc. Natl Acad. Sci. USA 109(26), 1782-1790 (2012).

17. Claessens A, Adams Y, Ghumra A et al. A subset of group A-like var genes encodes the malaria parasite ligands for binding to human brain endothelial cells. Proc. Natl Acad. Sci. USA 109(26), 1772-1781 (2012).

18. Lavstsen T, Turner L, Saguti F et al. Plasmodium falciparum erythrocyte membrane protein 1 domain cassettes 8 and 13 are associated with severe malaria in children. Proc. Natl Acad. Sci. USA 109(26), 1791-1800 (2012).

19. Rowe JA, Claessens A, Corrigan RA, Arman M. Adhesion of Plasmodium falciparuminfected erythrocytes to human cells: molecular mechanisms and therapeutic implications. Expert Rev. Mol. Med. 11, e16 (2009).

20. Hanson J, Lam SW, Mahanta KC et al. Relative contributions of macrovascular and microvascular dysfunction to disease severity in falciparum malaria. J. Infect. Dis. 206(4), 571-579 (2012).

21. Schofield L, Hackett F. Signal transduction in host cells by a glycosylphosphatidylinositol toxin of malaria parasites. J. Exp. Med. 177(1), 145-153 (1993).
22. Barrera V, Skorokhod OA, Baci D, Gremo G, Arese P, Schwarzer E. Host fibrinogen stably bound to hemozoin rapidly activates monocytes via TLR- 4 and CD11b/CD18integrin: a new paradigm of hemozoin action. Blood 117(21), 5674-5682 (2011).

23. Shio MT, Kassa FA, Bellemare MJ, Olivier $M$. Innate inflammatory response to the malarial pigment hemozoin. Microbes Infect. 12(12-13), 889-899 (2010).

24. Prato M, D’Alessandro S, Van den Steen PE et al. Natural haemozoin modulates matrix metalloproteinases and induces morphological changes in human microvascular endothelium. Cell Microbiol. 13(8), 1275-1285 (2011).

25. Medana IM, Turner GD. Plasmodium falciparum and the blood-brain barrier - contacts and consequences. J. Infect. Dis. 195(7), 921-923 (2007).

26. Brown $\mathrm{H}$, Rogerson $\mathrm{S}$, Taylor $\mathrm{T}$ et al. Blood-brain barrier function in cerebral malaria in Malawian children. Am. J. Trop. Med. Hyg. 64(3-4), 207-213 (2001).

27. Dorovini-Zis K, Schmidt K, Huynh $\mathrm{H}$ et al. The neuropathology of fatal cerebral malaria in malawian children. Am. J. Pathol. 178(5), 2146-2158 (2011).

28. Francischetti IM, Seydel KB, Monteiro RQ et al. Plasmodium falciparum-infected erythrocytes induce tissue factor expression in endothelial cells and support the assembly of multimolecular coagulation complexes. J. Thromb. Haemost. 5(1), 155-165 (2007).

29. Newton CR, Kirkham FJ, Winstanley PA et al. Intracranial pressure in African children with cerebral malaria. Lancet 337(8741), 573-576 (1991).

30. Newton CR, Peshu N, Kendall B et al. Brain swelling and ischaemia in Kenyans with cerebral malaria. Arch. Dis. Child 70(4), 281-287 (1994).

31. Potchen MJ, Kampondeni SD, Seydel KB et al. Acute brain MRI findings in 120 Malawian children with cerebral malaria: new insights into an ancient disease. AJNR Am. J. Neuroradiol. 33(9), 1740-1746 (2012).

32. Bottius E, Guanzirolli A, Trape JF, Rogier C, Konate L, Druilhe P. Malaria: even more chronic in nature than previously thought; evidence for subpatent parasitaemia detectable by the polymerase chain reaction. Trans. $R$. Soc. Trop. Med. Hyg. 90(1), 15-19 (1996).

33. Mackenzie S. Retinal haemorrhages and melanemia as symptoms of ague. Med. Times Gazette 662-665 (1877).

34. Lewallen S, Bakker H, Taylor TE, Wills BA, Courtright P, Molyneux ME. Retinal findings predictive of outcome in cerebral malaria. Trans. R. Soc. Trop. Med. Hyg. 90(2), 144-146 (1996).
35. Beare NA, Glover SJ, Molyneux M. Malarial retinopathy in cerebral malaria. Am. J. Trop. Med. Hyg. 80(2), 171 (2009).

36. Seydel KB, Fox LL, Glover SJ et al. Plasma concentrations of parasite histidine-rich protein 2 distinguish between retinopathypositive and retinopathy-negative cerebral malaria in Malawian children. J. Infect. Dis. 206(3), 309-318 (2012).

37. Taylor T. Caring for children with cerebral malaria: insights gleaned from 20 years on a research ward in Malawi. Trans. R. Soc. Trop. Med. Hyg. 103(Suppl. 1), S6-S10 (2009).

38. Miller LH, Su X. Artemisinin: discovery from the Chinese herbal garden. Cell 146(6), 855-858 (2011).

39. Kyu HH, Fernandez E. Artemisinin derivatives versus quinine for cerebral malaria in African children: a systematic review. Bull. World Health Organ. 87(12), 896-904 (2009).

40. Dondorp AM, Fanello CI, Hendriksen IC et al. Artesunate versus quinine in the treatment of severe falciparum malaria in African children (AQUAMAT): an openlabel, randomised trial. Lancet 376(9753), 1647-1657 (2010).

41. Hess KM, Goad JA, Arguin PM. Intravenous artesunate for the treatment of severe malaria. Ann. Pharmacother. 44(7-8), 1250-1258.

42. Dondorp AM, Nosten F, Yi P et al. Artemisinin resistance in Plasmodium falciparum malaria. N. Engl. J. Med. 361(5), 455-467 (2009).

43. Phyo AP, Nkhoma S, Stepniewska K et al. Emergence of artemisinin-resistant malaria on the western border of Thailand: a longitudinal study. Lancet 379(9830), 1960-1966 (2012).

44. Dondorp A, Nosten F, Stepniewska K, Day $\mathrm{N}$, White N. Artesunate versus quinine for treatment of severe falciparum malaria: a randomised trial. Lancet 366(9487), 717-725 (2005).

45. Maitland K, Pamba A, Newton CR, Levin M. Response to volume resuscitation in children with severe malaria. Pediatr. Crit. Care Med. 4(4), 426-431 (2003).

46. Maitland K, Levin M, English $M$ et al. Severe P. falciparum malaria in Kenyan children: evidence for hypovolaemia. QJM96(6), 427-434 (2003).

47. Akech S, Ledermann H, Maitland K. Choice of fluids for resuscitation in children with severe infection and shock: systematic review. BMJ341, c4416 (2010).

48. Maitland K, Kiguli S, Opoka RO et al. Mortality after fluid bolus in African children with severe infection. N. Engl. J. Med. 364(26), 2483-2495 (2011). 
49. English M, Wale S, Binns G, Mwangi I, Sauerwein H, Marsh K. Hypoglycaemia on and after admission in Kenyan children with severe malaria. QJM91(3), 191-197 (1998).

50. Ogetii GN, Akech S, Jemutai J et al. Hypoglycaemia in severe malaria, clinical associations and relationship to quinine dosage. BMC Infect. Dis. 10, 334 (2010).

51. Idro R, Carter JA, Fegan G, Neville BG, Newton CR. Risk factors for persisting neurological and cognitive impairments following cerebral malaria. Arch. Dis. Child. 91(2), 142-148 (2006).

52. Graz B, Dicko M, Willcox ML et al. Sublingual sugar for hypoglycaemia in children with severe malaria: a pilot clinical study. Malar. J. 7, 242 (2008).

53. Phiri K, Esan M, van Hensbroek MB, Khairallah C, Faragher B, ter Kuile FO. Intermittent preventive therapy for malaria with monthly artemether-lumefantrine for the post-discharge management of severe anaemia in children aged 4-59 months in southern Malawi: a multicentre, randomised, placebocontrolled trial. Lancet Infect. Dis. 12(3), 191-200 (2012).

54. Obonyo CO, Vulule J, Akhwale WS, Grobbee DE. In-hospital morbidity and mortality due to severe malarial anemia in western Kenya. Am. J. Trop. Med. Hyg. 77(6 Suppl.), S23-S28 (2007).

55. Bojang KA, Palmer A, Boele van Hensbroek M, Banya WA, Greenwood BM. Management of severe malarial anaemia in Gambian children. Trans. R. Soc. Trop. Med. Hyg. 91(5), 557-561 (1997).

56. Schwartz E. Iron deficiency anemia. In: Nelson's Textbook of Pediatrics. WB Saunders (Ed.). Elsevier Health Sciences, PA, USA, 1387-1389 (1996).

57. Meremikwu M, Smith HJ. Blood transfusion for treating malarial anaemia. Cochrane Database Syst. Rev. 2, CD001475 (2000).

58. WHO. Global blood safety initiative. Guidelines for appropriate use of blood. WHO, Geneva, Switzerland (1989).

59. Idro R, Gwer S, Kahindi M et al. The incidence, aetiology and outcome of acute seizures in children admitted to a rural Kenyan district hospital. BMC Pediatr. 8, 5 (2008).

60. Ogutu BR, Newton CR, Crawley J et al. Pharmacokinetics and anticonvulsant effects of diazepam in children with severe falciparum malaria and convulsions. Br. J. Clin. Pharmacol. 53(1), 49-57 (2002).

61. Cunnington AJ, de Souza JB, Walther M, Riley EM. Malaria impairs resistance to Salmonella through heme- and heme oxygenase-dependent dysfunctional granulocyte mobilization. Nat. Med. 18(1), 120-127 (2012).

62. MacLennan CA. Host defense against malaria favors Salmonella. Nat. Med. 18(1), 21-22 (2012).

63. Bronzan RN, Taylor TE, Mwenechanya J et al. Bacteremia in Malawian children with severe malaria: prevalence, etiology, HIV coinfection, and outcome. J. Infect. Dis. 195(6), 895-904 (2007).

64. Nadjm B, Amos B, Mtove G et al. WHO guidelines for antimicrobial treatment in children admitted to hospital in an area of intense Plasmodium falciparum transmission: prospective study. BMJ340, c1350 (2010).

65. Were T, Davenport GC, Hittner JB et al. Bacteremia in Kenyan children presenting with malaria. J. Clin. Microbiol. 49(2), 671-676 (2011).

66. Mpimbaza A, Staedke SG, Ndeezi G, Byarugaba J, Rosenthal PJ. Predictors of anticonvulsant treatment failure in children presenting with malaria and prolonged seizures in Kampala, Uganda. Malar. J. 8, 145 (2009).

67. Marik PE, Zaloga GP. Early enteral nutrition in acutely ill patients: a systematic review. Crit. Care Med. 29(12), 2264-2270 (2001).

68. Maude RJ, Hoque G, Hasan MU et al. Timing of enteral feeding in cerebral malaria in resource-poor settings: a randomized trial. PLoS One 6(11), e27273 (2011).

69. Bruzzone R, Dubois-Dalcq M, Grau GE, Griffin DE, Kristensson K. Infectious diseases of the nervous system and their impact in developing countries. PLoS Pathog. 5(2), e1000199 (2009).

70. Enwere G. A review of the quality of randomized clinical trials of adjunctive therapy for the treatment of cerebral malaria. Trop. Med. Int. Health 10(11), 1171-1175 (2005).

71. Crawley J, Waruiru C, Mithwani S et al. Effect of phenobarbital on seizure frequency and mortality in childhood cerebral malaria: a randomised, controlled intervention study. Lancet 355(9205), 701-706 (2000).

72. Warrell DA, Looareesuwan S, Warrell MJ et al. Dexamethasone proves deleterious in cerebral malaria. A double-blind trial in 100 comatose patients. N. Engl. J. Med.306(6), 313-319 (1982).

73. Hoffman SL, Rustama D, Punjabi NH et al. High-dose dexamethasone in quinine-treated patients with cerebral malaria: a double-blind, placebo-controlled trial. J. Infect. Dis. 158(2), 325-331 (1988).

74. Hoffman SL, Punjabi NH, Kumala S et al. Reduction of mortality in chloramphenicoltreated patients with severe typhoid fever by high dose dexamethasone. Trans. Assoc. Am. Phys. 96, 188-196 (1983).

75. Grau GE, Craig AG. Cerebral malaria pathogenesis: revisiting parasite and host contributions. Future Microbiol. 7(2), 291-302 (2012).

76. Grau GE, Fajardo LF, Piguet PF, Allet B, Lambert PH, Vassalli P. Tumor necrosis factor (cachectin) as an essential mediator in murine cerebral malaria. Science 237(4819), 1210-1212 (1987).

77. Kwiatkowski D, Hill AV, Sambou I et al. TNF concentration in fatal cerebral, non-fatal cerebral, and uncomplicated Plasmodium falciparum malaria. Lancet 336(8725), 1201-1204 (1990).

78. Grau GE, Taylor TE, Molyneux ME et al. Tumor necrosis factor and disease severity in children with falciparum malaria. N. Engl. J. Med.320(24), 1586-1591 (1989).

79. van Hensbroek MB, Palmer A, Onyiorah E et al. The effect of a monoclonal antibody to tumor necrosis factor on survival from childhood cerebral malaria. J. Infect. Dis. 174(5), 1091-1097 (1996).

80. Conroy AL, Glover SJ, Hawkes M et al. Angiopoietin-2 levels are associated with retinopathy and predict mortality in Malawian children with cerebral malaria: a retrospective case-control study*. Crit. Care Med. 40(3), 952-959 (2012).

81. Lopansri BK, Anstey NM, Weinberg JB et al. Low plasma arginine concentrations in children with cerebral malaria and decreased nitric oxide production. Lancet 361(9358), 676-678 (2003).

82. Serghides L, Kim H, Lu Z et al. Inhaled nitric oxide reduces endothelial activation and parasite accumulation in the brain, and enhances survival in experimental cerebral malaria. PLoS One 6(11), e27714 (2011).

83. Bergmark B, Bergmark R, De Beaudrap P et al. Inhaled nitric oxide and cerebral malaria: basis of a strategy for buying time for pharmacotherapy. Pediatr. Infect. Dis. J. doi:10.1097/INF.0b013e318266c113 (2012) (Epub ahead of print).

84. Hawkes M, Opoka RO, Namasopo S et al. Inhaled nitric oxide for the adjunctive therapy of severe malaria: protocol for a randomized controlled trial. Trials 12, 176 (2011).

85. Clark TD, Njama-Meya D, Nzarubara B et al. Incidence of malaria and efficacy of combination antimalarial therapies over 4 years in an urban cohort of Ugandan children. PLoS One 5(7), el1759 (2010).

86. Boivin MJ, Bangirana P, Byarugaba J et al. Cognitive impairment after cerebral malaria in children: a prospective study. Pediatrics 119(2), e360-e366 (2007). 
87. Dugbartey AT, Spellacy FJ, Dugbartey MT. Somatosensory discrimination deficits following pediatric cerebral malaria. Am. J. Trop. Med. Hyg. 59(3), 393-396 (1998).

88. Dugbartey AT. The neuropsychology of cerebral malaria. In: Unpublished Doctoral Dissertation. University of Victoria, Victoria, BC, Canada (1995).

89. Crawley J, Smith S, Muthinji P, Marsh K, Kirkham F. Electroencephalographic and clinical features of cerebral malaria. Arch. Dis. Child. 84(3), 247-253 (2001).

90. Idro R, Kakooza-Mwesige A, Balyejjussa S et al. Severe neurological sequelae and behaviour problems after cerebral malaria in Ugandan children. BMC Res. Notes 3, 104 (2010).

91. Magen J, Kauye F, Vokhiwa M, SemrudClikeman M, Taylor T, Boivin M. Adapting the Achenbach child behavior checklist $(\mathrm{CBCL})$ as a psychiatric screening tool for DSM IV diagnosis in Malawian children. Presented at: Malawi Psychiatric Research and Intervention Conference. Blantyre, Malawi, 7-8 February 2011.

92. Gamanagatti S, Kandpal H. MR imaging of cerebral malaria in a child. Eur. J. Radiol. 60(1), 46-47 (2006).

93. Fayed N, Modrego PJ, Castillo J, Davila $\mathrm{J}$. Evidence of brain dysfunction in attention deficit-hyperactivity disorder: a controlled study with proton magnetic resonance spectroscopy. Acad. Radiol. 14(9), 1029-1035 (2007).

94. Idro R, Jenkins NE, Newton CR. Pathogenesis, clinical features, and neurological outcome of cerebral malaria. Lancet Neurol. 4(12), 827-840 (2005).

95. Rubia K, Smith AB, Brammer MJ, Taylor E. Temporal lobe dysfunction in medicationnaive boys with attention-deficit/hyperactivity disorder during attention allocation and its relation to response variability. Biol. Psychiatry 62(9), 999-1006 (2007).

96. Smith AB, Taylor E, Brammer M, Toone B, Rubia K. Task-specific hypoactivation in prefrontal and temporoparietal brain regions during motor inhibition and task switching in medication-naive children and adolescents with attention deficit hyperactivity disorder. Am. J. Psychiatry 163(6), 1044-1051 (2006).

97. Banaschewski T, Hollis C, Oosterlaan J et al. Towards an understanding of unique and shared pathways in the psychopathophysiology of ADHD. Dev. Sci. 8(2), 132-140 (2005).

98. Jung SL, Kim BS, Lee KS, Yoon KH, Byun JY. Magnetic resonance imaging and diffusion-weighted imaging changes after hypoglycemic coma. J. Neuroimaging 15(2), 193-196 (2005).

99. Yamamoto T, Shibata N, Muramatsu F, Sakayori N, Kobayashi M. Oxidative stress in the human fetal brain: an immunohistochemical study. Pediatr. Neurol. 26(2), 116-122 (2002).

100. Krishnamoorthy KS, Soman TB, Takeoka M, Schaefer PW. Diffusion-weighted imaging in neonatal cerebral infarction: clinical utility and follow-up. J. Child Neurol. 15(9), 592-602 (2000).

101. John CC, Panoskaltsis-Mortari A, Opoka RO et al. Cerebrospinal fluid cytokine levels and cognitive impairment in cerebral malaria. Am. J. Trop. Med. Hyg. 78(2), 198-205 (2008).

102. Clark IA, Alleva LM, Vissel B. The roles of TNF in brain dysfunction and disease. Pharmacol. Ther. 128(3), 519-548 (2010).

103. Bangirana P, Giordani B, John CC, Page C, Opoka RO, Boivin MJ. Immediate neuropsychological and behavioral benefits of computerized cognitive rehabilitation in Ugandan pediatric cerebral malaria survivors. J. Dev. Behav. Pediatr. 30(4), 310-318 (2009).

104. Boivin MJ. Effects of early cerebral malaria on cognitive ability in Senegalese children. J. Dev. Behav. Pediatr. 23(5), 353-364 (2002).

105. Carter JA, Mung'ala-Odera V, Neville BG et al. Persistent neurocognitive impairments associated with severe falciparum malaria in Kenyan children. J. Neurol. Neurosurg. Psychiatry 76(4), 476-481 (2005).

106. Carter JA, Ross AJ, Neville BG et al. Developmental impairments following severe falciparum malaria in children. Trop. Med. Int. Health 10(1), 3-10 (2005).

107. Snow RW, Guerra CA, Noor AM, Myint HY, Hay SI. The global distribution of clinical episodes of Plasmodium falciparum malaria. Nature 434(7030), 214-217 (2005).

108. Snow RW, Newton CRJC, Craig MH, Steketee RW. The public health burden of Plasmodium falciparum malaria in Africa: deriving the numbers. Disease Control Priorities Project, working paper no. 11 (2003).

109. Holding PA, Stevenson J, Peshu N, Marsh K. Cognitive sequelae of severe malaria with impaired consciousness. Trans. R. Soc. Trop. Med. Hyg. 93(5), 529-534 (1999).

110. Holding PA, Taylor HG, Kazungu SD et al. Assessing cognitive outcomes in a rural African population: development of a neuropsychological battery in Kilifi District, Kenya. J. Int. Neuropsychol. Soc. 10(2), 246-260 (2004).
111. Holding PA, Kitsao-Wekulo PK. Describing the burden of malaria on child development: what should we be measuring and how should we be measuring it? Am. J. Trop. Med. Hyg. 71(Suppl. 2), S71-S79 (2004).

112. Opoka RO, Bangirana P, Boivin MJ, John CC, Byarugaba J. Seizure activity and neurological sequelae in Ugandan children who have survived an episode of cerebral malaria. Afr. Health Sci. 9(2), 75-81 (2009).

113. Jukes MC, Pinder M, Grigorenko EL et al. Long-term impact of malaria chemoprophylaxis on cognitive abilities and educational attainment: follow-up of a controlled trial. PLoS Clin. Trials 1(4), e19 (2006).

114. Clarke SE, Jukes MC, Njagi JK et al. Effect of intermittent preventive treatment of malaria on health and education in schoolchildren: a cluster-randomised, double-blind, placebocontrolled trial. Lancet 372(9633), 127-138 (2008).

115. Abubakar A, Van De Vijver FJ, Mithwani S et al. Assessing developmental outcomes in children from Kilifi, Kenya, following prophylaxis for seizures in cerebral malaria. J. Health Psychol. 12(3), 417-430 (2007).

116. Boivin M, Giordani B. Neuropsychological assessment of African children: evidence for a universal basis to cognitive ability. In: Cultural Neuroscience: Cultural Influences on Brain Function. Chiao J (Ed.). Elsevier Publications, NY, USA, 113-135 (2009).

117. Mendis K, Rietveld A, Warsame M, Bosman A, Greenwood B, Wernsdorfer WH. From malaria control to eradication: the WHO perspective. Trop. Med. Int. Health 14(7), 802-809 (2009).

118. Lindsay SW, Gibson ME. Bednets revisited - old idea, new angle. Parasitol. Today 4(10), 270-272 (1988).

119. Alonso PL, Lindsay SW, Armstrong JR et al. The effect of insecticide-treated bed nets on mortality of Gambian children. Lancet 337(8756), 1499-1502 (1991).

120. Greenwood BM, Greenwood AM, Bradley AK et al. Comparison of two strategies for control of malaria within a primary health care programme in the Gambia. Lancet 1(8595), 1121-1127 (1988).

121. Aponte JJ, Schellenberg D, Egan A et al. Efficacy and safety of intermittent preventive treatment with sulfadoxine-pyrimethamine for malaria in African infants: a pooled analysis of six randomised, placebocontrolled trials. Lancet 374(9700), 1533-1542 (2009).

122. Schellenberg JR, Maokola W, Shirima K et al. Cluster-randomized study of intermittent preventive treatment for malaria 
in infants (IPTi) in southern Tanzania: evaluation of impact on survival. Malar. J. 10, 387 (2011).

123. WHO policy recommendation on Intermittent Preventive Treatment during infancy with sulphadoxine-pyrimethamine (IPTi) for Plasmodium falciparum malaria control in Africa. WHO, Geneva, Switzerland (2010).

124. Meremikwu MM, Donegan S, Sinclair D, Esu E, Oringanje C. Intermittent preventive treatment for malaria in children living in areas with seasonal transmission. Cochrane Database Syst. Rev. 2, CD003756 (2012).

125. Clyde DF, McCarthy VC, Miller RM, Hornick RB. Specificity of protection of man immunized against sporozoite-induced falciparum malaria. Am. J. Med. Sci. 266(6), 398-403 (1973).

126. WHO. Initiative for vaccine research: malaria vaccines. WHO, Geneva, Switzerland (2012).
127. Agnandji ST, Lell B, Soulanoudjingar SS et al. First results of phase 3 trial of RTS,S/ AS01 malaria vaccine in African children. N. Engl. J. Med. 365(20), 1863-1875 (2011).

128. Thera MA, Plowe CV. Vaccines for malaria: how close are we? Annu. Rev. Med. 63, 345-357 (2012).

129. Epstein JE, Tewari K, Lyke KE et al. Live attenuated malaria vaccine designed to protect through hepatic CD8 ${ }^{+} \mathrm{T}$ cell immunity. Science 334(6055), 475-480 (2011). 


\section{$\underset{\text { EDUCATION }}{\text { Medlscape Pediatric cerebral malaria: a scourge of Africa }}$}

To obtain credit, you should first read the journal article. After reading the article, you should be able to answer the following, related, multiple-choice questions. To complete the questions (with a minimum $70 \%$ passing score) and earn continuing medical education (CME) credit, please go to www.medscape.org/journal/fnl. Credit cannot be obtained for tests completed on paper, although you may use the worksheet below to keep a record of your answers. You must be a registered user on Medscape.org. If you are not registered on Medscape.org, please click on the New Users: Free Registration link on the left hand side of the website to register. Only one answer is correct for each question. Once you successfully answer all post-test questions you will be able to view and/or print your certificate. For questions regarding the content of this activity, contact the accredited provider,
CME@medscape.net. For technical assistance, contactCME@webmd.net. American Medical Association's Physician's Recognition Award (AMA PRA) credits are accepted in the US as evidence of participation in CME activities. For further information on this award, please refer to http://www.ama-assn.org/ama/pub/ category $/ 2922 . h t m l$. The AMA has determined that physicians not licensed in the US who participate in this CME activity are eligible for AMA PRA Category 1 Credits $^{\mathrm{TM}}$. Through agreements that the AMA has made with agencies in some countries, AMA PRA credit may be acceptable as evidence of participation in CME activities. If you are not licensed in the US, please complete the questions online, print the AMA PRA CME credit certificate and present it to your national medical association for review.

\section{Activity evaluation: where 1 is strongly disagree and 5 is strongly agree.}

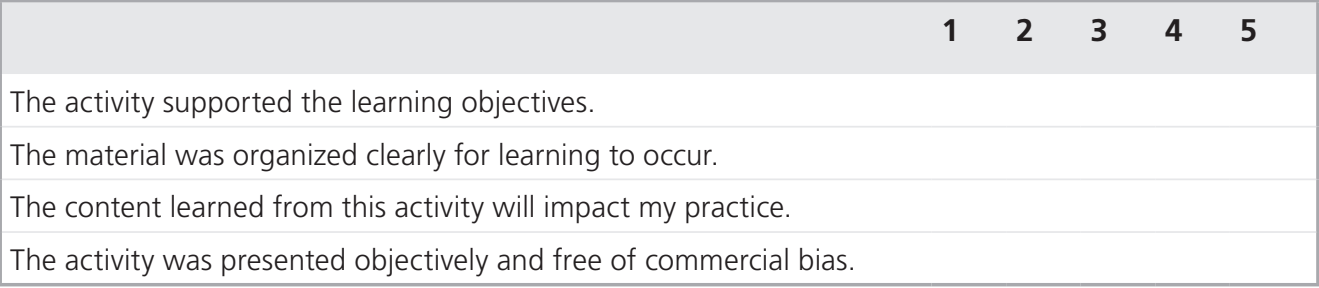

1. Your patient is a 3-year-old girl with coma and Plasmodium falciparum parasitemia. Based on the review by Dr. Postels and colleagues, which of the following statements about diagnosis and outcomes of pediatric cerebral malaria is most likely correct?

$\square$ A The presence of malarial retinopathy is $95 \%$ sensitive and $100 \%$ specific for cerebral parasite sequestration in cerebral vasculature

B The World Health Organization (WHO) clinical definition of cerebral malaria (CM) is highly specific

C With optimal treatment, mortality rate is about $2 \%$

D With optimal treatment, about $10 \%$ of survivors have neurological complications

2. Based on the review by Dr. Postels and colleagues, which of the following statements about treatment of $\mathrm{CM}$ for the patient described in question 1 is most likely correct?
A Treatment with artemisinin derivatives is not recommended
B Supportive care, including detection and treatment of hypoglycemia, seizures, and anemia, is extremely important
C Phenobarbital has been proven effective as adjuvant therapy
D The role of anti-TNF $\alpha$ antibodies in treatment is well defined 
3. Based on the review by Dr. Postels and colleagues, which of the following statements about the epidemiology and prevention of pediatric CM would most likely be correct?
A The incidence of malaria is increasing throughout sub-Saharan Africa
B Treatment of active malaria is a more effective and important strategy than malaria prevention
C Bed nets, chemoprophylaxis, and vaccines are all important in malaria control and elimination programs
D Malaria vaccines are proven to protect completely against all forms of malarial disease

